\title{
Delineating the Efficacy of a Cannabis-Based Medicine at Advanced Stages of Dementia in a Murine Model
}

\author{
Ester Aso $^{\mathrm{a}, \mathrm{b}, *}$, Pol Andrés-Benito ${ }^{\mathrm{a}, \mathrm{b}}$ and Isidro Ferrer ${ }^{\mathrm{a}, \mathrm{b}}$ \\ a Institut de Neuropatologia, Servei d'Anatomia Patològica, IDIBELL-Hospital Universitari de Bellvitge, \\ Universitat de Barcelona, L'Hospitalet de Llobregat, Spain \\ ${ }^{\mathrm{b}}$ CIBERNED, Centro de Investigación Biomédica en Red de Enfermedades Neurodegenerativas, \\ Instituto Carlos III, Spain
}

Handling Associate Editor: Tommaso Cassano

Accepted 19 June 2016

\begin{abstract}
Previous reports have demonstrated that the combination of $\Delta^{9}$-tetrahydrocannabinol ( $\Delta^{9}$-THC) and cannabidiol (CBD) botanical extracts, which are the components of an already approved cannabis-based medicine, reduce the Alzheimerlike phenotype of A $\beta P P / P S 1$ transgenic mice when chronically administered during the early symptomatic stage. Here, we provide evidence that such natural cannabinoids are still effective in reducing memory impairment in A $\beta P P / P S 1$ mice at advanced stages of the disease but are not effective in modifying the $A \beta$ processing or in reducing the glial reactivity associated with aberrant $A \beta$ deposition as occurs when administered at early stages of the disease. The present study also demonstrates that natural cannabinoids do not affect cognitive impairment associated with healthy aging in wild-type mice. The positive effects induced by $\Delta^{9}$-THC and CBD in aged A $\beta P P / P S 1$ mice are associated with reduced GluR2/3 and increased levels of GABA-A R $\alpha 1$ in cannabinoid-treated animals when compared with animals treated with vehicle alone.
\end{abstract}

Keywords: Advanced stages, Alzheimer's disease, cannabidiol, $\Delta^{9}$-tetrahydrocannabinol, dementia

\section{INTRODUCTION}

Dementia is a general term referring to a progressive decline in cognitive abilities occurring usually in the elderly due to a variety of causes. Among the symptoms, memory loss, language impairment, attention deficiencies, and difficulty in reasoning and judgment are the most prominent. Etiologies of

\footnotetext{
${ }^{*}$ Correspondence to: Ester Aso, Institut de Neuropatologia, Servei d'Anatomia Patològica, IDIBELL-Hospital Universitari de Bellvitge, C/Feixa Llarga s/n, 08907 L'Hospitalet de Llobregat, Spain. Tel.: +34 93 2607452; Fax: +34 93 2607503; E-mail: aso@bellvitgehospital.cat.
}

dementia may be divided into two broad categories: (i) degenerative, reflecting pathological processes which are intrinsic to the central nervous system and usually involve aberrant protein processing, and (ii) non-degenerative, which include acquired or secondary dementias linked to vascular, endocrine, traumatic, and other primary central and systemic diseases. Alzheimer's disease (AD) is the most common type of dementia, accounting for 60 to $80 \%$ of cases, and is characterized by the presence in the brain of extracellular deposits of amyloid- $\beta(A \beta)$, a peptide derived from the aberrant processing of the transmembrane amyloid- $\beta$ protein precursor $(\mathrm{A} \beta \mathrm{PP})$, 
and intracellular neurofibrillary tangles composed of hyperphosphorylated microtubule-associated protein tau. $\mathrm{AD}$ is also associated with neuroinflammation and oxidative stress, two pathological processes that exacerbate neurodegeneration during $\mathrm{AD}$ progression $[1,2]$.

Recent studies have demonstrated the therapeutic effects of several compounds acting on the endocannabinoid system in neurodegenerative diseases such as AD [3-6]. The endocannabinoid system is a complex network of cellular receptors and signaling molecules [7] highly expressed in brain and targeted by cannabis derivatives which, when activated, provides neuroprotection by reducing neuronal damage, neuroinflammation, and oxidative stress, as well as promoting intrinsic repair mechanisms [3]. Thus, chronic stimulation with selective synthetic agonists of $\mathrm{CB}_{1}$ and $\mathrm{CB}_{2}$ receptors, the most well-known cannabinoid receptors, reduces cognitive impairment and brain alterations associated with $A \beta$ production, in at least three different animal models of AD [8-11]. Moreover, the combination of $\Delta^{9}$-tetrahydrocannabinol $\left(\Delta^{9}\right.$-THC) and cannabidiol (CBD), two phytocannabinoids produced by the plant Cannabis sativa, reduces the pathological phenotype in mouse models of $\mathrm{AD}$ and tauopathy when administered at early stages of the disease $[12,13]$. These natural compounds are the two main components of Sativex ${ }^{\circledR}$, which is a well-tolerated medicine prescribed for the treatment of spasticity associated with multiple sclerosis.

The aim of the present study was to broaden our knowledge about the potential beneficial effect of the $\Delta^{9}$-THC and CBD combination in reducing dementia symptoms at advanced stages of the disease. The present study was designed to evaluate the effect of this cannabis-based medicine administered in old A $\beta P P / P S 1$ mice and in aged wild-type littermates. Our results may contribute to increased understanding of the possible use of the $\Delta^{9}$-THC and CBD combination in demented patients.

\section{MATERIALS AND METHODS}

\section{Animals}

The experiments were carried out in male A $\beta P P / P S 1$ mice and wild-type-like (WT) littermates aged 12 months at the onset of the study and in male WT mice aged 3 months (non-aged controls) with a C57B16J genetic background. The generation of mice expressing the human mutated A $\beta P P$ swe and
PS1dE9 has been described elsewhere [14]. All the animals used in this study derived from 7 breeder pairs. Animals were maintained under standard animal housing conditions (static isolation caging, 3-4 animals per cage) in a 12-h dark-light cycle with free access to food and water. Mice were randomly assigned to treatment groups and the experiments were conducted under blind conditions. All animal procedures were carried out following the guidelines of the European Communities Council Directive 2010/63/EU and with the approval of the local ethical committee of the University of Barcelona.

\section{Pharmacological treatment}

$\Delta^{9}$-THC-enriched botanical extract (containing $67.0 \% \Delta^{9}$-THC, $0.8 \%$ CBD, $1.2 \%$ cannabigerol, $0.9 \%$ cannabichromene and $3.2 \%$ other phytocannabinoids) and CBD-enriched botanical extract (containing $62.7 \%$ CBD, $3.6 \% \Delta^{9}$-THC, $1.4 \%$ cannabigerol, $5.7 \%$ cannabichromene and $1.8 \%$ other phytocannabinoids) were supplied by GW Pharmaceuticals Ltd (Cambridge, UK). The extracts $\left(\Delta^{9}\right.$-THC $0.75 \mathrm{mg} / \mathrm{kg}+\mathrm{CBD} 0.75 \mathrm{mg} / \mathrm{kg}$ ) were dissolved in 5\% ethanol, $5 \%$ Tween, and $90 \%$ saline, and this combination was administered intraperitoneally (i.p.) in a single injection in a volume of $10 \mathrm{~mL} / \mathrm{kg}$ body weight. The human equivalent dose (HED) calculated with the formula for dose translation based on body surface area [15] corresponds to $0.04 \mathrm{mg} / \mathrm{kg}$ for each cannabinoid, which is equivalent to the administration of a single Sativex ${ }^{\circledR}$ oromucosal spray $(2.8 \mathrm{mg}$ $\Delta^{9}-\mathrm{THC}+2.8 \mathrm{mg}$ CBD) in a human being weighing $70 \mathrm{~kg}$. At this dose, the compound lacks psychoactivity. Groups of animals were treated once a day for 5 weeks with the extracts or with vehicle alone. The number of animals included in each group was as follows: WT 12 months treated with cannabinoids, $n=9$; vehicle alone, $n=8$; A $\beta P P / P S 112$ months with cannabinoids, $n=11$; A $\beta P P / P S 112$ months treated with vehicle alone, $n=10$; WT aged 3 months treated with cannabinoids, $n=8$; treated with vehicle alone, $n=7$. After a 10-day washing period, animals were subjected to behavioral evaluation.

\section{Cognitive evaluation and sample collection}

Memory performance was evaluated with the twoobject recognition test. On day 1 , mice were placed for 9 min in a V-maze, in which two identical objects were situated at the ends of the arms; the time that the mice spent exploring each object was recorded. 
Then, $24 \mathrm{~h}$ after the training session, animals were placed again for $9 \mathrm{~min}$ in the $\mathrm{V}$-maze, with one of the two familiar objects replaced by a novel object. The time that the animals spent exploring the two objects was recorded and an object recognition index (RI) was calculated, as the difference between the time spent exploring the novel object $\left(\mathrm{T}_{\mathrm{N}}\right)$ and the familiar object $\left(\mathrm{T}_{\mathrm{F}}\right)$ divided by the total time spent exploring the two objects $\left[R I=\left(T_{N}-T_{F}\right) /\left(T_{N}+T_{F}\right)\right]$.

At the end of the behavioral testing, the animals were killed and their brains rapidly removed from the skull and processed for study. One hemisphere was dissected on ice, immediately frozen, and stored at $-80^{\circ} \mathrm{C}$ until use. The other hemisphere was fixed in $4 \%$ paraformaldehyde and processed for immunohistochemistry.

\section{A $\beta$ immunohistochemistry}

Fixed tissue samples were embedded in paraffin, and coronal sections, $4 \mathrm{~mm}$ thick, were cut with a microtome. Consecutive de-waxed sections were incubated with $98 \%$ formic acid (3 min) and then treated with citrate buffer $(20 \mathrm{~min})$ to enhance antigenicity. Then endogenous peroxidases were blocked by incubation in $10 \%$ methanol- $1 \% \mathrm{H}_{2} \mathrm{O}_{2}$ solution $(15 \mathrm{~min})$. Sections were blocked with $3 \%$ normal horse serum solution and then incubated at $4{ }^{\circ} \mathrm{C}$ overnight with the primary antibody against total A $\beta$ (clone 6F/3D 1:50, Dako, Glostrup, Denmark), $A \beta_{40}$ (1:100, Merck Millipore, Billerica, MA, USA) or $A \beta_{42}(1: 50$, Merck Millipore). Sections were subsequently rinsed and incubated with biotinylated secondary antibodies (Dako). Peroxidase reaction was visualized with diaminobenzidine and $\mathrm{H}_{2} \mathrm{O}_{2}$. Sections were slightly counterstained with hematoxylin. After staining, the sections were dehydrated and cover-slipped for observation under a Nikon Eclipse E800 microscope (Nikon Imaging Inc., Tokyo, Japan). The cortical total $A \beta$ burden was calculated as the percentage of the area of amyloid deposition in plaques with respect to the total cortical area $\left(0.6 \mathrm{~mm}^{2}\right)$ in 9 pictures taken from 3 different sections $(-0.1 \mathrm{~mm},-1.5 \mathrm{~mm}$, and $-2.0 \mathrm{~mm}$ from bregma) of the each animal brain ( 3 pictures per section corresponding to cingular/retrospenial and motor cortex, somatosensory cortex, and piriform/entorhinal cortex). The areas selected were the main regions of the cerebral cortex in which $\mathrm{A} \beta$ is deposited in A $\beta \mathrm{PP} / \mathrm{PS} 1$ mice. In addition, two sections of the hippocampus of each animal $(-1.5 \mathrm{~mm}$ and $-2.0 \mathrm{~mm}$ from bregma) were used for quantification of $A \beta$ burden in the hippocampus, calculated as the percentage of the amyloid deposition in plaques with respect to the total hippocampal area in each section. The percentage of $A \beta_{42}$ contents in each plaque was calculated by comparing the specific $A \beta_{42}$ staining with respect to total $A \beta_{42}+A \beta_{40}$ staining in at least 25 cortical plaques per animal in consecutive sections. A researcher who did not know the treatment received in each group performed the quantifications. $A \beta$ quantification was calculated using the Analysis tool of the Adobe ${ }^{\circledR}$ Photoshop $^{\circledR}$ CS4 software (Adobe Systems Inc., San Jose, CA, USA). All the A $\beta P P / P S 1-t r e a t e d$ animals were analyzed.

\section{A $\beta$ soluble quantification: enzyme-linked immunosorbent assay (ELISA)}

Fresh-frozen mouse brain cortex was homogenized in 4 volumes (wt:vol) of TBS extraction buffer $(140 \mathrm{mM} \mathrm{NaCl}, 3 \mathrm{mM} \mathrm{KCl}, 25 \mathrm{mM}$ Tris $(\mathrm{pH}$ 7.4), $5 \mathrm{mM}$ EDTA, and protease inhibitor cocktail (Roche Molecular Systems, Pleasanton, CA, USA). Homogenate was spun at $100,000 \mathrm{~g}$ for $1 \mathrm{~h}$, and the supernatant was saved as the soluble fraction for $A \beta$ quantification. $A \beta_{40}$ and $A \beta_{42}$ Human ELISA kits (Invitrogen ${ }^{\mathrm{TM}}$ Corporation, Camarillo, CA, USA) were used to quantify the levels of $A \beta_{40}$ and $A \beta_{42}$ peptides in the brain soluble fractions. Quantitative determination was carried out according to the manufacturer's instructions. $A \beta_{40}$ and $A \beta_{42}$ levels were normalized to the total amount of protein from each individual sample (BCA method, Thermo Fisher Scientific, Wilmington, DE, USA). All treated animals were analyzed but only A $\beta P P / P S 1$ mice were used for quantification because, as expected, no specific immunolabeling was observed in any WT mice.

\section{Double-labeling immunofluorescence}

De-waxed sections were incubated with $98 \%$ formic acid (3 min) for $A \beta$ immunofluorescence and then treated with citrate buffer $(20 \mathrm{~min})$ to enhance antigenicity. Sections were stained with a saturated solution of Sudan black B for 30 min (Merck Millipore) to block lipofuscin autofluorescence, rinsed in $70 \%$ ethanol and washed in distilled water. After a blockade with $10 \%$ fetal bovine serum ( $90 \mathrm{~min}$ ), the sections were incubated at $4{ }^{\circ} \mathrm{C}$ overnight with combinations of primary antibodies against $A \beta$ (clone 6F/3D 1:50, Dako), glial fibrillary acidic protein (GFAP; $1: 250$, Dako) and IBA1 $(1: 250$, Wako, 
Richmond, VA, USA). After washing, the sections were incubated with Alexa488 and Alexa546 fluorescence secondary antibodies against the corresponding host species (1: 400, Molecular Probes, Eugene, OR, USA). The sections were washed and mounted in Immuno-Fluore Mounting medium (ICN Biomedicals, Solon, OH, USA), sealed, dried overnight and examined with a Nikon Eclipse E800 microscope. The specific GFAP and IBA1 immunostaining density was calculated in reference to the $A \beta$ plaque area in 5 representative pictures taken from the cortex of each animal using the Adobe ${ }^{\circledR}$ Photoshop ${ }^{\circledR}$ CS4 software. All the A $\beta P P / P S 1-$ treated animals were used for quantifications.

\section{Gel electrophoresis and western blotting}

Frozen samples of the somatosensory cortex were homogenized in RIPA lysis buffer $(50 \mathrm{mM}$ Tris/HCl buffer, pH 7.4 containing $2 \mathrm{mM}$ EDTA, $0.2 \%$ Nonidet P-40, $1 \mathrm{mM}$ PMSF, protease, and phosphatase inhibitor cocktails, Roche Molecular Systems, USA). The homogenates were centrifuged for $15 \mathrm{~min}$ at 13,000 rpm. Protein concentration was determined with the BCA method (Thermo Scientific). Equal amounts of protein $(20 \mu \mathrm{g})$ for each sample were loaded and separated by electrophoresis on sodium dodecyl sulfate polyacrylamide gel electrophoresis (SDS-PAGE) (10\%) gels and transferred onto nitrocellulose membranes (Amersham, Freiburg, Germany). Non-specific bindings were blocked by incubation in 3\% albumin in PBS containing $0.2 \%$ Tween for $1 \mathrm{~h}$ at room temperature. After washing, membranes were incubated overnight at $4{ }^{\circ} \mathrm{C}$ with the antibodies against thioredoxin 2 (Txn2, $13 \mathrm{kDa}$, rabbit, 1:1,000, Proteintech, Chicago, IL, USA), wingless-related integration site 16 (Wnt16, $37 \mathrm{kDa}$, rabbit, $1: 5,000$, GeneTex, Irvine, CA, USA), synaptosome associated protein $25 \mathrm{kDa}$ (SNAP25, $25 \mathrm{kDa}$, mouse, 1:1,000, Abcam, Cambridge, UK), postsynaptic density protein $95 \mathrm{kDa}$ (PSD-95, $95 \mathrm{kDa}$, rabbit, 1:1,000; Merck Millipore), synaptotagmin (47 kDa, mouse, $1: 1,000$; Abcam), glutamate receptor 2/3 (GluR2/3, $110 \mathrm{kDa}$, rabbit, Merck Millipore), and gamma-aminobutyric acid receptor A subunit $\alpha 1$ (GABA-A R $\alpha 1,51 \mathrm{kDa}$, rabbit, Santa Cruz Biotechnology, Dallas, TX, USA). Protein loading was monitored using an antibody against $\beta$-tubulin (50 kDa, $1: 10,000$, Abcam). Membranes were then incubated for $1 \mathrm{~h}$ in the appropriate HRP-conjugated secondary antibodies $(1: 2,000$, Dako), and immunocomplexes were revealed by chemiluminescence reagent (ECL, Amersham). Densitometric quantification was carried out with TotalLab v2.01 software (Pharmacia, Sweden). Bands were normalized to $\beta$-tubulin. Six animals per group were analyzed.

\section{Statistical analysis}

Statistical analysis was performed with the SPSS ${ }^{\circledR}$ Statistics v21.0 software (IBM, New York, NY, USA). The normality of the data was assessed with the Shapiro-Wilk test, and parametric statistical tests were used for the analysis of all the data in the study. Memory data were analyzed with two-way ANOVA by group (depending on age and genotype) and treatment, followed by Tukey's post hoc. Protein quantifications with western blotting were analyzed using two-way ANOVA with genotype and treatment as between factors. $A \beta$ and glia quantifications were analyzed with Student's $t$-test. In all the experiments, the significance level was set at $p<0.05$.

\section{RESULTS}

Natural cannabinoids reduce memory deficits in A $B P P / P S 1$ mice at advanced stages of AD-like pathology but have no beneficial effects in aged WT mice

Daily administration of $\Delta^{9}$-THC and CBD $(0.75 \mathrm{mg} / \mathrm{kg}$ each botanical extract i.p.) for 5 weeks at advanced stages of the symptomatic phase (12 months) blunted the memory impairment observed in vehicle-treated A $\beta P P / P S 1$ mice, as revealed by the two-object recognition test (Fig. 1). Two-way ANOVA revealed a significant group $\left(F_{(2,39)}=7.579, p<0.01\right)$ and treatment effect $\left(F_{(1,39)}=4.840, p<0.05\right)$ and interaction between the two factors $\left(F_{(2,39)}=6.810, p<0.01\right)$. Subsequent Tukey's post hoc tests revealed that vehicle-treated WT mice aged 12 months $(p<0.05)$ and vehicletreated A $\beta P P / P S 1$ mice aged 12 months $(p<0.001)$ exhibited memory impairment when compared to corresponding non-aged control mice. Interestingly, $\Delta^{9}$-THC + CBD significantly increased the recognition index of 12-month-old A $\beta P P / P S 1$ mice when compared to vehicle-treated littermates $(p<0.001)$. However, the treatment was not effective in reducing the memory impairment exhibited by 12-month-old WT mice. Treated mice were also tested in the active avoidance test, which is a more complex task and involves both memory and learning processes. However, aged WT mice evidenced difficulties to perform 


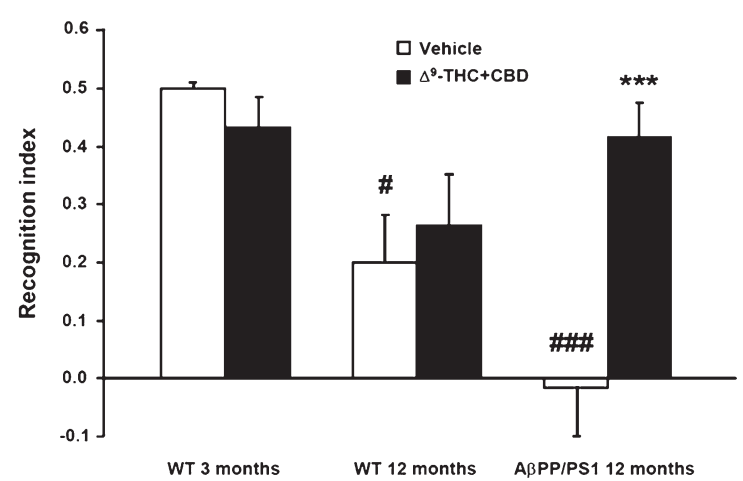

Fig. 1. Memory performance of animals in the two-object recognition test. WT and AßPP/PS1 mice aged 12 months at the beginning of the study exhibit a significant reduction in the recognition index when compared with non-aged control mice (3 months old WT mice). Daily administration of $\Delta^{9}-\mathrm{THC}+\mathrm{CBD}(0.75 \mathrm{mg} / \mathrm{kg}$ each botanical extract i.p.) for 5 weeks blunts memory impairment of $\mathrm{A} \beta \mathrm{PP} / \mathrm{PS} 1$ mice at advanced stages (12 months). However, it is not effective in reducing memory impairment in 12-month-old WT littermates. Data are expressed as the mean values \pm SEM; ${ }^{* * *} p<0.001$ treatment effect; ${ }^{\#} p<0.05,{ }^{\# \# \#} p<0.001$ compared to vehicle-treated non-aged control mice.

this test, thus invalidating the possibility to distinguish cognitive performance between genotypes (data not shown).

The combination of $\triangle^{9}$-THC and CBD does not alter $A \beta$ processing in $A \beta P P / P S 1$ mice at advanced stages of the disease

Chronic treatment with the combination of $\Delta^{9}$ THC + CBD did not significantly modify total $A \beta$ burden in the neocortex and hippocampus of A $\beta$ PP/PS1 mice (Fig. 2A, B). In contrast to what was previously observed at early stages of AD-like pathology in A $\beta P P / P S 1$ mice [11], $\Delta^{9}-\mathrm{THC}+\mathrm{CBD}$ was not able to reduce the level of soluble $A \beta_{42}$ and $\mathrm{A} \beta_{40}$ when administered at advanced stages of the disease (Fig. 2E). In the same line, natural cannabinoids failed to induce a change in the composition of $\mathrm{A} \beta$ plaques similar to that observed when treatment was applied at early stages [13]. Despite an upward trend $(p=0.119)$, the percentage of $A \beta_{42}$ in each plaque was not significantly increased in treated mice (Fig. 2C, D). None of the assessed A $\beta$ forms was detectable in WT littermates (data not shown).

\section{Gliosis related to $A \beta$ deposition was not modified in A $\beta P P / P S 1$ mice treated at advanced stages}

In contrast to our previous observations showing reduced astroglial and microglial reactivity in the vicinity of $A \beta$ deposits in $A \beta P P / P S 1$ mice treated with $\Delta^{9}-\mathrm{THC}+\mathrm{CBD}$ during the early symptomatic stages [13], the present results show that the same combination of natural cannabinoids was not able to reduce glial reactivity in A $\beta P P / P S 1$ mice when administered at advanced stages of the disease (Fig. 3).

\section{$\triangle^{9}$-THC and CBD combination modifies the levels of several synaptic and neurotransmission markers}

In order to evaluate the possible molecular mechanisms underlying the beneficial effects of $\Delta^{9}$-THC + $\mathrm{CBD}$ on $\mathrm{A} \beta \mathrm{PP} / \mathrm{PS} 1$ mice at advanced stages, we analyzed, using western blotting, selected proteins linked to redox metabolism, and synapses and neurotransmission in cortical homogenates (Fig. 4). First, we evaluated the levels of the redox protein Txn2 and the signaling protein Wnt16. However, two-way ANOVA revealed no significant effect of treatment independently of genotype, and no interaction between the two factors on the levels of Txn2 or Wnt16 in cortical homogenates of A $\beta P P / P S 1-m i c e$ treated at advanced stages. Next, we evaluated the levels of certain proteins involved in the regulation of synaptic plasticity and function, including SNAP25, PSD95 and synaptotagmin. $\Delta^{9}-$ THC + CBD induced an effect on the levels of SNAP25 (Genotype effect: $F_{(1,18)}=0.61, N . S$.; Treatment effect: $F_{(1,18)}=7.07$, $p<0.05$; Interaction: $F_{(1,18)}=3.08$, N.S.). Thus, chronic treatment reduced the amounts of SNAP25 protein in A $\beta P P / P S 1$ mice, which were elevated in comparison to WT littermates, when compared to A $\beta P P / P S 1$ mice treated with vehicle alone $(p<0.05)$. In contrast, cannabinoids did not reduce the elevated levels of PSD-95 observed in A $\beta P P / P S 1$ mice (Genotype effect: $F_{(1,19)}=6.03, p<0.05$; Treatment effect: $F_{(1,19)}=0.01, N . S$; Interaction: $F_{(1,19)}=0.29$, N.S.). When compared to WT mice, the increase in PSD-95 only reached statistical significance in $\Delta^{9}$-THC + CBD-treated A $\beta P P / P S 1$ mice $(p<0.05)$, although a clear tendency was also observed in vehicle-treated A $\beta P P / P S 1$ mice. Two-way ANOVA revealed no significant effect of treatment or genotype, nor interaction between the two factors, at the level of synaptotagmin in treated mice. Finally, we evaluated the protein levels of the metabotropic glutamate receptor $2 / 3(\mathrm{GluR} 2 / 3)$ and the ionotropic GABA-A receptor $\alpha 1$. Two-way ANOVA revealed that chronic treatment significantly modulated both GluR2/3 (Genotype effect: $F_{(1,19)}=16.492$, 


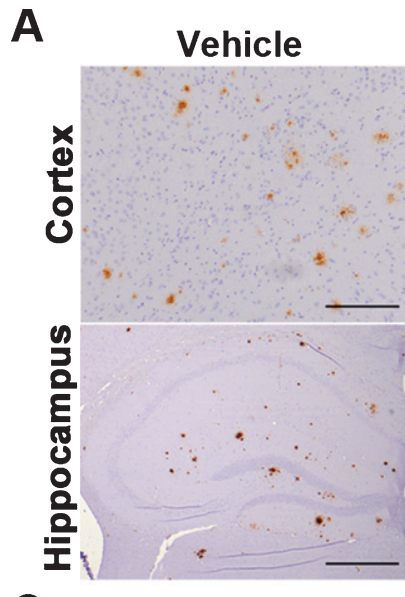

$\Delta^{9}-\mathrm{THC}+\mathrm{CBD}$

B
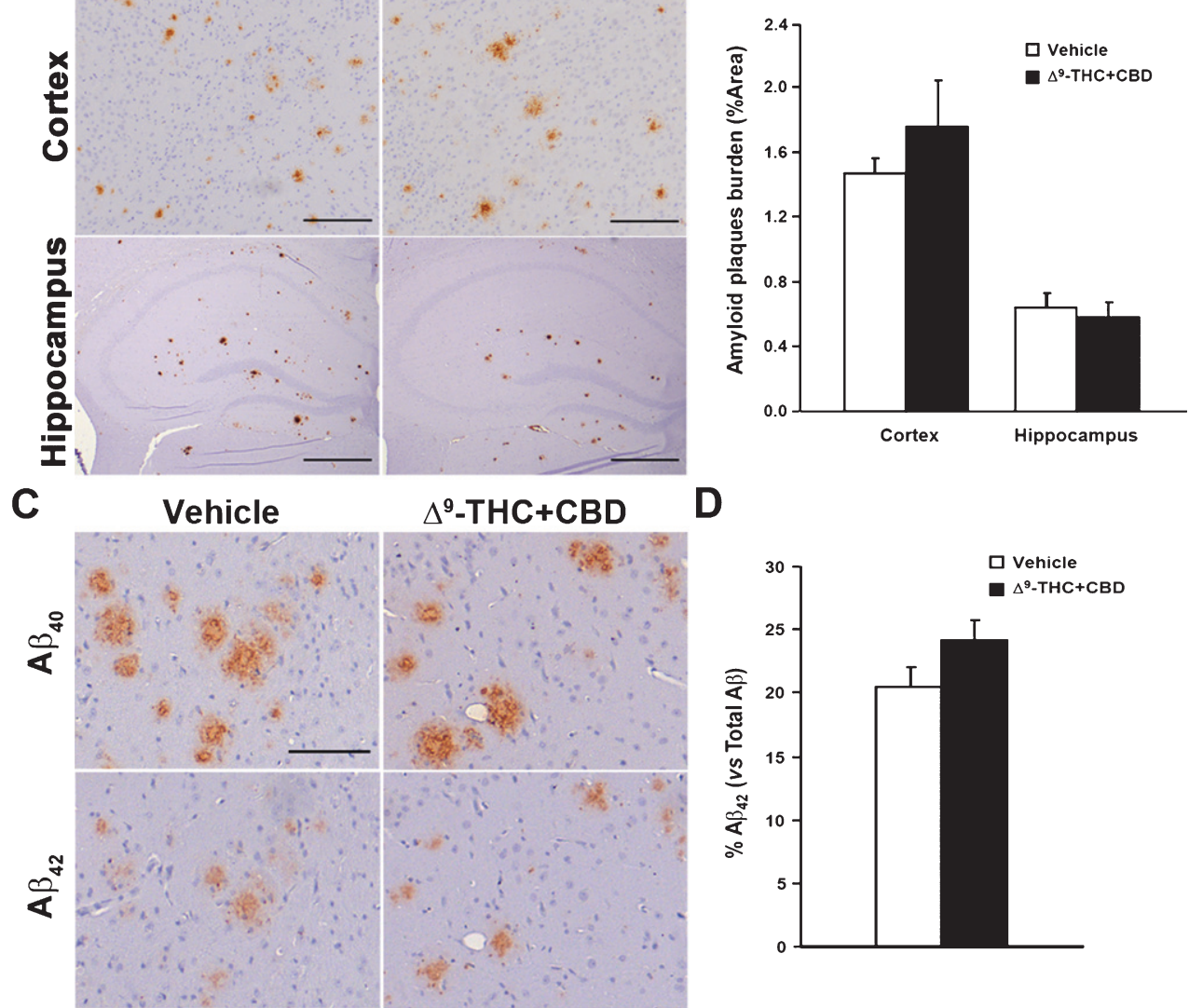

E

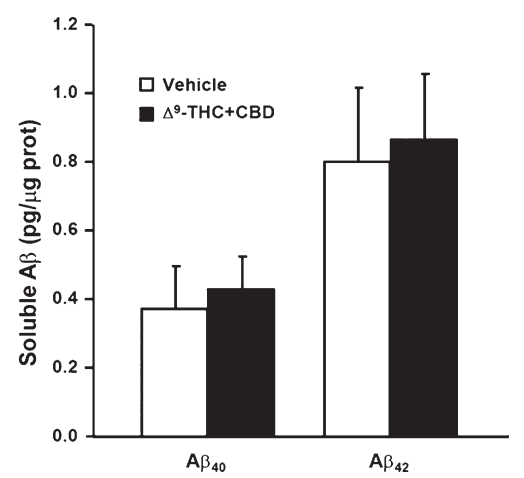

Fig. 2. A) Representative images of the $A \beta$ immunoreactivity in somatosensory cortex (top) and hippocampus (bottom) in vehicle- (left) and $\Delta^{9}$-THC + CBD-treated (right) A $\beta P P / P S 1$ mice. Scale bars represent $200 \mu \mathrm{m}$ (cortex) or $500 \mu \mathrm{m}$ (hippocampus). B) Total A $\beta$ burden quantification reveals no treatment effect in cortex and hippocampus in aged A $\beta P P / P S 1$ mice. C) Representative images of the A $\beta_{40}$ (top) and $A \beta_{42}$ (bottom) immunoreactivity in consecutive cortical sections of vehicle- (left) and $\Delta^{9}$-THC + CBD-treated (right) A $\beta P P / P S 1$ mice aged 12 months at the beginning of the study. Scale bar represents $50 \mu \mathrm{m}$. D) Quantification of the percentage of A $\beta_{42}$ contents with respect to total $A \beta_{42}+A \beta_{40}$ levels in each plaque reveals no significant effect in spite of a tendency toward increase in $\Delta^{9}$-THC + CBD-treated mice. E) The combination of $\Delta^{9}$-THC + CBD does not modify soluble $A \beta_{40}$ and $A \beta_{42}$ levels in cortical homogenates from A $\beta P P / P S 1$ mice treated at advanced stages of the disease. Counts are expressed as the mean values \pm SEM.

$p<0.001$; Treatment effect: $F_{(1,19)}=0.09$, N.S.; Interaction: $\left.F_{(1,19)}=7.09, p<0.05\right)$ and GABA-A $\mathrm{R} \alpha 1$ receptors (Genotype effect: $F_{(1,19)}=0.23$, N.S.;
Treatment effect: $F_{(1,19)}=1.93$, N.S.; Interaction: $\left.F_{(1,19)}=8.93, p<0.01\right)$. Thus, subsequent post hoc tests showed that $\Delta^{9}$-THC + CBD significantly 
A
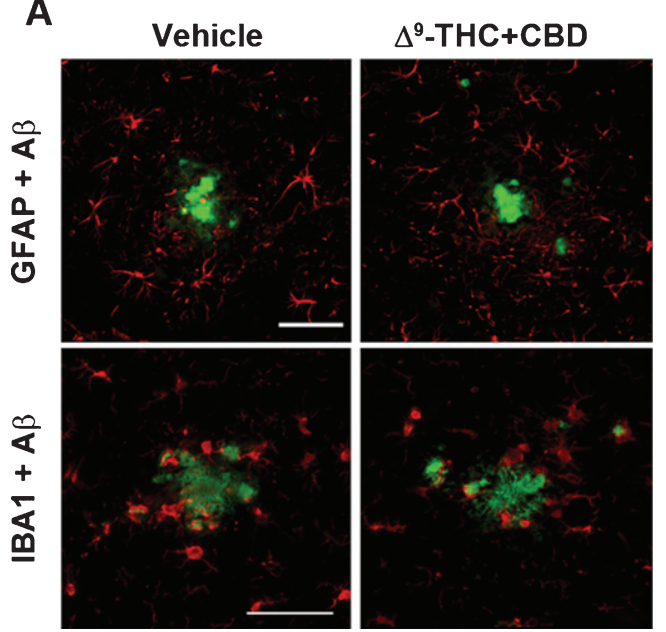

B

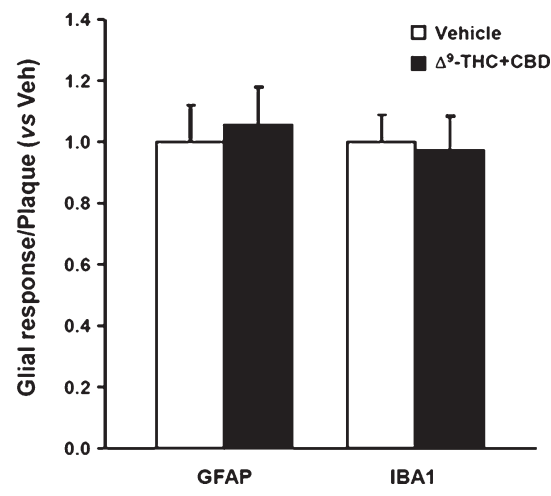

Fig. 3. A) Representative images of double GFAP (red, upper panels) and IBA1 (red, lower panels) and A $\beta$ (green) immunoreactivity in sections of the neocortex of AßPP/PS1 mice chronically treated with natural cannabinoids at the age of 12 months. Scale bars represent $50 \mu \mathrm{m}$. B) Quantification of the GFAP and IBA1 staining around A $\beta$ plaques reveals no significant effect of the treatment on astrocytes and microglia in A $\beta P P / P S 1$ mice when administered at advanced stages of the disease. Data are expressed as the mean values \pm SEM.

reduced the levels of GluR2/3 in A $\beta P P / P S 1$ mice compared to vehicle-treated $(p<0.05)$ and WT littermates $(p<0.01)$, and increased the GABA-A R $\alpha 1$ levels with respect to vehicle-treated A $\beta P P / P S 1$ mice $(p<0.05)$, which exhibited reduced levels compared to WT animals $(p<0.05)$.

\section{DISCUSSION}

During the last decade, targeting of the endogenous cannabinoid system has emerged as a promising therapy against Alzheimer-type dementia because endocannabinoid signaling modulates some pathological processes linked to neurodegenerative processes, including protein misfolding, neuroinflammation, excitotoxicity, mitochondrial dysfunction, and oxidative stress [3-6]. However, most evidence is based on observations in experimental models at early stages of the disease. The present study analyzes the efficacy of a cannabis-based medicine, composed essentially of $\Delta^{9}$-THC and CBD, at advanced stages of Alzheimertype dementia in A $\beta P P / P S 1$ transgenic mice. Collaterally, we evaluated the therapeutic profile of the $\Delta^{9}$-THC and CBD combination in WT littermates used as a model of aging. We chose the combination of these two natural cannabinoids because, although previous findings revealed that both $\Delta^{9}$-THC and CBD given alone resulted in beneficial cognitive effects in A $\beta P P / P S 1$ mice $[13,16,17]$, the combination of $\Delta^{9}$-THC and CBD was more effective than either cannabinoid alone in these transgenic animals when administered at early stages [13].

The present results show that treatment with $\Delta^{9}$ THC and CBD at non-psychoactive doses reduces memory impairment occurring in A $\beta P P / P S 1$ mice at 12 months of age when the progression of the disease is at an advanced stage. These findings might appear to contradict the evidence about the cognitive impairment induced by $\Delta^{9}$-THC exposure in healthy mice [18]. However, the doses employed revealing memory impairments in mice [18] are much higher than the dose used here showing a neuroprotective effect. Second, the brain context of young healthy mice exposed to high doses of $\Delta^{9}$-THC is different from that of aged AD mice, since molecular reorganization of the endogenous cannabinoid system and altered neuronal signaling are found in AD [19]. These differences may explain different $\Delta^{9}$-THClinked effects in these two groups of mice. Finally, the combination of natural cannabinoids administered in the present study is composed of $\Delta^{9}$-THC in a similar proportion to $\mathrm{CBD}$, which is known to antagonize $\Delta^{9}$-THC-induced deficits in memory [20].

The $\Delta^{9}$-THC- and CBD-enriched extract combination administered at advanced stages of the disease does not modify $A \beta$ burden or the glial reactivity associated with aberrant $A \beta$ deposition in $A \beta P P / P S 1$ mice, in contrast to the effect when administered at early stages of the disease [13]. Thus, these findings suggest that natural cannabinoids modulate mechanisms other than $A \beta$ processing and glial reactions 


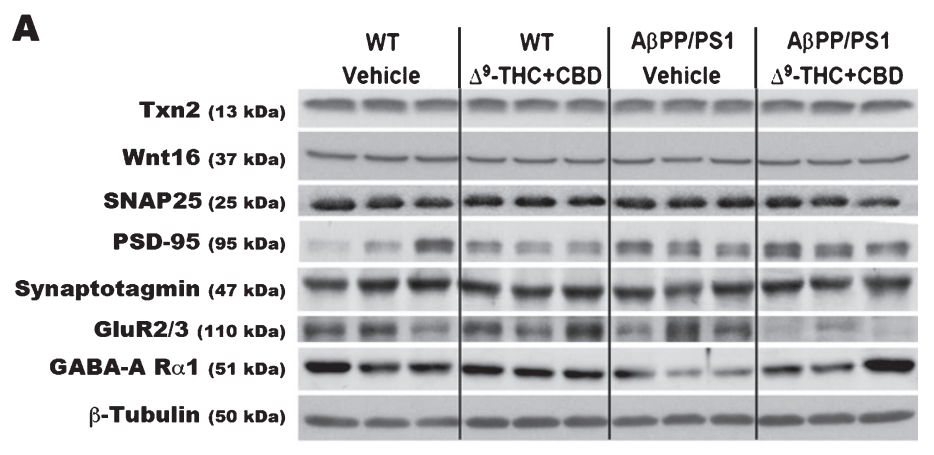

B

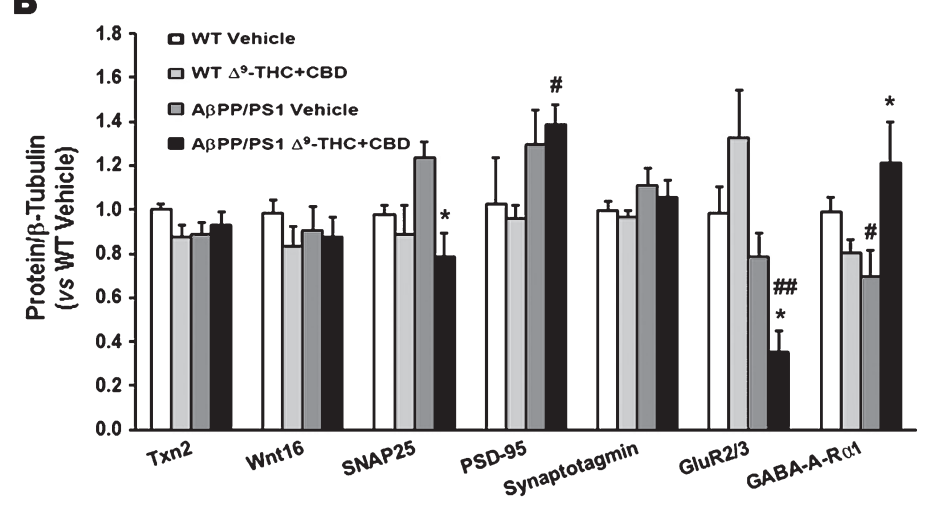

Fig. 4. Western blot quantification of proteins in somatosensory cortex homogenates in WT and A $\beta P P / P S 1$-treated mice with cannabinoids or with vehicle alone: thioredoxin 2 (Txn2), wingless-related integration site (Wnt16), synaptosome associated protein-25 (SNAP25), post-synaptic density protein-95 (PSD-95), synaptotagmin, glutamate receptor 2/3 (GluR2/3) and gamma-aminobutyric acid receptor A $\alpha 1$ (GABA-A R $\alpha 1$ ). A) Representative immunoblots for Txn2, Wnt16, SNAP25, PSD-95, synaptotagmin, GluR2/3, GABA-A R $\alpha 1$, and corresponding $\beta$-tubulin loading control indicating the molecular weight of proteins. B) Optical density quantification of each immunoblot with respect to WT-vehicle group which is considered as control. No significant change in the total amount of Txn2 or Wnt 16 is observed in any group. However, the $\Delta^{9}-\mathrm{THC}+\mathrm{CBD}$ combination normalizes SNAP25 levels but fails to reduce levels of PSD-95 or to modify synaptotagmin protein levels. $\Delta^{9}$-THC + CBD modifies the expression of certain neurotransmission-related molecules. Thus, A $\beta P P / P S 1-t r e a t e d$ mice exhibit significantly lower levels of GluR2/3 and increased levels of GABA-A R $\alpha 1$ protein when compared with vehicle-treated transgenic mice. Densitometric quantifications are expressed as mean values \pm SEM. ${ }^{*} p<0.05$ treatment effect. ${ }^{\#} p<0.05$, ${ }^{\# \#} p<0.01$, genotype effect.

which contribute to cognitive improvement revealed in A $\beta P P / P S 1$ mice treated at advanced stages of the disease. We may speculate that the mechanisms involved in this cannabinoid-induced beneficial effect on memory performance are specific for neurodegenerative dementia since the $\Delta^{9}$-THC and CBD combination is ineffective at reducing mild cognitive impairment in old WT littermates used as a model of behavior deterioration linked to old age.

Among the potential molecular mechanisms involved in the beneficial effects induced by the $\Delta^{9}$-THC and CBD combination in AßPP/PS1 mice treated at advanced stages of the disease, we focused our attention on evaluating three groups of proteins: (i) Txn2 and Wnt16, two proteins that play a role in the $\Delta^{9}$-THC + CBD-induced effects on A $\beta P P / P S 1$ mice when treated at early stages of the disease [13], (ii) SNAP25, which is associated with synaptosome and contributes to the formation of the exocytotic fusion complexes in neurons, PSD-95, located in the post-synaptic density of neurons and involved in receptors clustering, modulation of ion channels and associated signaling proteins, and synaptotagmin, a presynaptic $\mathrm{Ca}^{2+}$ sensor involved in the regulation of the traffic of synaptic vesicles and neurotransmitter release, as markers of synaptic plasticity and function, and (iii) GluR2/3 and GABA-A R $\alpha 1$, both involved in the regulation of neurotransmission in cortical brain areas. The selection of these markers was based on previous evidence about the role of cannabinoids in modulating synaptic signaling and function [21, 22]. In contrast to what happened when $\Delta^{9}$-THC and CBD were administered at early stages [13], the combination of these natural cannabinoids fails to modify the levels of the redox protein Txn2 and the signaling molecule Wnt16 in the somatosensory 
cortex of aged A $\beta P P / P S 1$ mice. However, the combination of $\Delta^{9}$-THC and CBD induces some changes in markers of synaptic function, which might be related to the cognitive improvement observed in A $\beta P P / P S 1$ mice treated during advanced stages of the disease. Thus, chronic treatment normalized the levels of the pre-synaptic SNAP25, but not the post-synaptic PSD-95 protein, in A $\beta P P / P S 1$ mice. Increased levels of synaptic proteins such as SNAP25 were previously associated with aberrant neuritic sprouting in brain areas affected by $A \beta$ synthesis and deposition in $\mathrm{AD}$ [23], suggesting that $\Delta^{9}$-THC and CBD might contribute to reducing this pathological process. More importantly, A $\beta P P / P S 1$ mice treated with $\Delta^{9}$-THC and CBD at advanced stages exhibit a reduction in GluR2/3 expression levels accompanied by an increase in the expression of GABA-A R $\alpha 1$. These results suggest that natural cannabinoids alter the imbalance of excitatory versus inhibitory neural activity in the somatosensory cortex of aged A $\beta P P / P S 1$ mice [24, 25]. In fact, $A \beta$ has been demonstrated to inhibit long-term plasticity processes associated with learning and memory through suppression of $\mathrm{CB}_{1}$-dependent GABAergic synaptic disinhibition [26]. Moreover, $\mathrm{CB}_{1}$ receptor activity in GABAergic neurons protects against agedependent cognitive decline [27]. Therefore, it may be speculated that the combination of $\Delta^{9}$-THC and CBD facilitates inhibitory GABAergic activity in the somatosensory cortex by mitigating the deleterious effect of $\mathrm{A} \beta$ on GABAergic function, and subsequently on cognitive performance, via activation of $\mathrm{CB}_{1}$ receptors. $\Delta^{9}$-THC is also known to depress glutamate synaptic transmission via $\mathrm{CB}_{1}$ receptor activation, affecting glutamate release, inhibiting receptors and transporters function, reducing enzyme activity, and disrupting glutamate synaptic plasticity after prolonged exposure [28]. Thus, the reduction of GluR2/3 expression observed on treated mice might be the result of such depressed glutamatergic activity induced by chronic stimulation of $\mathrm{CB}_{1}$ receptor. These modifications could contribute to counteract the alterations in neural excitability observed in A $\mathrm{PPP} / \mathrm{PS} 1$ mice $[24,25]$ and in turn to improve cognitive performance. However, further experimental evidence will be needed to confirm the specific role of $\mathrm{CB}_{1}$ receptors in such effects.

In light of these observations, and of the fact that Sativex ${ }^{\circledR}$ is devoid of psychoactivity and is welltolerated at the dose proposed in our studies [29], potential translation to human beings might offer a safe and effective therapeutic agent in advanced Alzheimer-type dementia.

\section{ACKNOWLEDGMENTS}

We thank T. Yohannan for editorial help and GW Pharmaceuticals Ltd for the supply of the botanical extracts. This study was supported by grants from the CIBERNED and Instituto de Salud Carlos III, and co-funded by FEDER funds/European Regional Development Fund (ERDF)-a way to build Europe (PIE14/00034 and PI14/00757 to IF, and IFI15/00035 fellowship to PA-B).

Authors' disclosures available online (http://j-alz. com/manuscript-disclosures/16-0533r1).

\section{REFERENCES}

[1] Ferrer I (2012) Defining Alzheimer as a common age-related neurodegenerative process not inevitably leading to dementia. Prog Neurobiol 97, 38-51.

[2] Selkoe DJ (2012) Preventing Alzheimer's disease. Science 337, 1488-1492.

[3] Campbell VA, Gowran A (2007) Alzheimer's disease; taking the edge off with cannabinoids? Br J Pharmacol 152, 655-662.

[4] Koppel J, Davies P (2008) Targeting the endocannabinoid system in Alzheimer's disease. J Alzheimers Dis 15, 495504.

[5] Aso E, Ferrer I (2014) Cannabinoids for treatment of Alzheimer's disease: Moving toward the clinic. Front Pharmacol 5, 37.

[6] Bedse G, Romano A, Lavecchia AM, Cassano T, Gaetani S (2015) The role of endocannabinoid signaling in the molecular mechanisms of neurodegeneration in Alzheimer's disease. J Alzheimers Dis 43, 1115-1136.

[7] Lu HC, Mackie K (2016) An introduction to the endogenous cannabinoid system. Biol Psychiatry 79, 516-525.

[8] Aso E, Palomer E, Juvés S, Maldonado R, Muñoz FJ, Ferrer I (2012) $\mathrm{CB}_{1}$ agonist ACEA protects neurons and reduces the cognitive impairment of A $\beta P P / P S 1$ mice. J Alzheimers Dis 30, 439-459.

[9] Aso E, Juvés S, Maldonado R, Ferrer I (2013) $\mathrm{CB}_{2}$ cannabinoid receptor agonist ameliorates Alzheimer-like phenotype in AßPP/PS1 mice. J Alzheimers Dis 35, 847-858.

[10] Martín-Moreno AM, Brera B, Spuch C, Carro E, GarcíaGarcía L, Delgado M, Pozo MA, Innamorato NG, Cuadrado A, de Ceballos ML (2012) Prolonged oral cannabinoid administration prevents neuroinflammation, lowers $\beta$-amyloid levels and improves cognitive performance in Tg APP 2576 mice. J Neuroinflammation 9, 8.

[11] Wu J, Bie B, Yang H, Xu JJ, Brown DL, Naguib M (2013) Activation of the $\mathrm{CB}(2)$ receptor system reverses amyloidinduced memory deficiency. Neurobiol Aging 34, 791-804.

[12] Casarejos MJ, Perucho J, Gómez A, Muñoz MP, FernándezEstévez M, Sagredo O, Fernández Ruiz J, Guzmán M, de Yébenes JG, Mena MA (2013) Natural cannabinoids improve dopamine neurotransmission and tau and amyloid pathology in a mouse model of tauopathy. J Alzheimers Dis 35, 525-539. 
[13] Aso E, Sánchez-Pla A, Vegas-Lozano E, Maldonado R, Ferrer I (2015) Cannabis-based medicine reduces multiple pathological processes in A $\beta P P / P S 1$ mice. J Alzheimers Dis 43, 977-991.

[14] Borchelt DR, Ratovitski T, van Lare J, Lee MK, Gonzales V, Jenkins NA, Copeland NG, Price DL, Sisodia SS (1997) Accelerated amyloid deposition in the brains of transgenic mice coexpressing mutant presenilin 1 and amyloid precursor proteins. Neuron 19, 939-945.

[15] Reagan-Shaw S, Nihal M, Ahmad N (2008) Dose translation from animal to human studies revisited. FASEB J 22, 659661.

[16] Cheng D, Low JK, Logge W, Garner B, Karl T (2014) Chronic cannabidiol treatment improves social and object recognition in double transgenic APPswe/PS1 $\triangle \mathrm{E} 9$ mice. Psychopharmacology (Berl) 231, 3009-3017.

[17] Cheng D, Spiro AS, Jenner AM, Garner B, Karl T (2014) Long-term cannabidiol treatment prevents the development of social recognition memory deficits in Alzheimer's disease transgenic mice. J Alzheimers Dis 42:1383-1396.

[18] Chen R, Zhang J, Fan N, Teng ZQ, Wu Y, Yang H, Tang YP, Sun H, Song Y, Chen C (2013) $\Delta^{9}$-THC-caused synaptic and memory impairments are mediated through COX-2 signaling. Cell 155, 1154-1165.

[19] Mulder J, Zilberter M, Pasquare SJ, Alpar A, Schulte G, Ferreira SG, Kofalvi A, Martín-Moreno AM, Keimpema E, Tanila H,Watanabe M, Mackie K, Hortobagyi T, de Ceballos ML, Harkany T (2011) Molecular reorganization of endocannabinoid signalling in Alzheimer's disease. Brain 134, 1041-1060.

[20] Fadda P, Robinson L, Fratta W, Pertwee RG, Riedel G (2004) Differential effects of THC- or CBD-rich cannabis extracts on working memory in rats. Neuropharmacology 47, 1170-1179.
[21] Hoffman AF, Lupica CR (2013) Synaptic targets of $\Delta 9$ tetrahydrocannabinol in the central nervous system. Cold Spring Harb Perspect Med 3, 8.

[22] Castillo PE, Younts TJ, Chávez AE, Hashimotodani Y (2012) Endocannabinoid signaling and synaptic function. Neuron 76, 70-81.

[23] Ferrer I, Martí E, Tortosa A, Blasi J (1998) Dystrophic neurites of senile plaques are defective in proteins involved in exocytosis and neurotransmission. $J$ Neuropathol Exp Neurol 57, 218-225.

[24] Kellner V, Menkes-Caspi N, Beker S, Stern EA (2014) Amyloid- $\beta$ alters ongoing neuronal activity and excitability in the frontal cortex. Neurobiol Aging 35, 1982-1991.

[25] D'Amelio M, Rossini PM (2012) Brain excitability and connectivity of neuronal assemblies in Alzheimer's disease: From animal models to human findings. Prog Neurobiol 99, 42-60.

[26] Orr AL, Hanson JE, Li D, Klotz A, Wright S, Schenk D, Seubert P, Madison DV (2014) beta-amyloid inhibits E-S potentiation through suppression of cannabinoid receptor 1-dependent synaptic disinhibition. Neuron 82, 1334-1345.

[27] Albayram O, Alferink J, Pitsch J, Piyanova A, Neitzert K, Poppensieker K, Mauer D, Michel K, Legler A, Becker A, Monory K, Lutz B, Zimmer A, Bilkei-Gorzo A (2011) Role of $\mathrm{CB}_{1}$ cannabinoid receptors on GABAergic neurons in brain aging. Proc Natl Acad Sci U S A 108, 11256-11261.

[28] Colizzi M, McGuire P, Pertwee RG, Bhattacharyya S (2016) Effect of cannabis on glutamate signalling in the brain: A systematic review of human and animal evidence. Neurosci Biobehav Rev 64, 359-381.

[29] Wade DT, Collin C, Stott C, Duncombe P (2010) Metaanalysis of the efficacy and safety of Sativex (nabiximols), on spasticity in people with multiple sclerosis. Mult Scler 16, 707-714. 